\title{
Electron-phonon correlations on spin texture of gapped helical Dirac Fermions
}

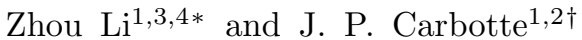 \\ 1 Department of Physics, McMaster University, Hamilton, Ontario Ontario, Canada,L8S $4 M 1$ \\ ${ }^{2}$ Canadian Institute for Advanced Research, Toronto, Ontario, Canada M5G $1 Z 8$ \\ ${ }^{3}$ Department of Physics and Astronomy, Louisiana State University, Baton Rouge, LA, 70803 USA \\ 4 Center for Computation and Technology, Louisiana State University, Baton Rouge, LA, 70803 USA
}

(Dated: May 30, 2022)

\begin{abstract}
The metallic surface states of a topological insulator support helical Dirac fermions protected by topology with their spin locked perpendicular to their momentum. They can acquire mass through magnetic doping or through hybridization of states on opposite faces of a thin sample. In this case there can be a component of electron spin oriented perpendicular to the surface plane. The electronphonon interaction renormalizes the dynamics of the charge carriers through their spectral density. It also modifies the gap channel and a second spectral function enters which, not only determines the out of plane spin component, but also comes into in-plane properties. While the out of plane spin component is decreased below the Fermi momentum $\left(k_{F}\right)$, the in plane component increases. There are also correlation tails extending well beyond $k_{F}$. The angular resolved photo-emission line shapes aquire Holstein side bands. The effective gap in the density of states is reduced and the optical conductivity aquires distinct measurable phonon structure even for modest value of the electron-phonon coupling.
\end{abstract}

PACS numbers: $75.70 \mathrm{Tj}, 71.38 \mathrm{Cn}, 78.67 .-\mathrm{n}$

\section{INTRODUCTION}

Helical Dirac fermions protected by topology and found at the surface of topological insulators $(\mathrm{TI})^{\underline{1}} \underline{\underline{\underline{4}}} \mathrm{ex}-$ hibit spin locked perpendicular to their momentum $\underline{\underline{4}-\underline{8}}$ Doping with magnetic impurities can break time reversal symmetry and create massive Dirac fermions as has been experimentally demonstrated ${ }^{9}$ in $\mathrm{Bi}_{2} \mathrm{Se}_{3}$. A gap can also be introduced in thin films when the distance between top and bottom surfaces is of the order of the extend in space of the surface states $\stackrel{10,11}{=}$ Gapped Dirac fermions arise in many other systems, for example in two dimensional membranes such as the dichalcoginide $\mathrm{MoS}_{2} \stackrel{12}{\underline{-16}}$ and silicene $\underline{\underline{17}} \underline{\underline{21}}$ with buckled honeycomb lattice. In both these cases pseudospin plays the analogous role to the real spin of topological insulators.

The electron-phonon interaction renormalizes quasiparticle dynamics and leads to important observable changes in electronic properties ${ }^{22}-31$ which illustrate the effects of many body renormalizations not captured in single particle theories. For the Dirac electrons in graphene as an example, features observed in the density of states $13-\underline{34}$ and in the dispersion curves measured in angular resolved photo emission spectroscopy ${ }^{35}$ have been interpreted as phonon structure ${ }^{36}$ The optical properties of graphene are also renormalized in a non trivial way $\stackrel{22,25,26,37,38}{ }$ In a simple bare band picture there is no optical absorption in the photon energy region between the Drude intraband contribution centered about $\omega=0$ and the interband onset at twice the value of the chemical potential $\omega=2 \mu$. In reality the real part of the dynamic longitudinal conductivity is observed to be finite and almost one third its universal background ${ }^{39.40}$ value in this photon region, ${ }^{37}$ This absorption is due to many body renormalizations and is at least partially assigned 22,26 to the electron-phonon interaction which provides boson assisted processes referred to as Holstein processes. A phonon is created along with an electron-hole pair.

Understanding the transport properties of the Dirac electrons on the surface of a topological insulator is important for possible device applications. At finite temperature the electron-phonon interaction is expected to be an important scattering chane ${ }^{41}, \mathrm{X}$. Zhu et al $\stackrel{42}{4}$ have studied the surface phonons on the (001) surface of $\mathrm{Bi}_{2} \mathrm{Se}_{3}$ and in particular have found a giant Kohn anomaly associated with a branch having a maximum of $18 \mathrm{THz}$. From measurements of the phonon self energy, the same group $\underline{43}$ determined the size of the electronphonon interaction report a coupling constant of 0.43 for a particular branch which is much larger than reported in angular resolved photo-emission spectroscopy. One such study by Z. H. Pan et al $\stackrel{44}{=}$ gives a mass enhancement $\lambda$ of

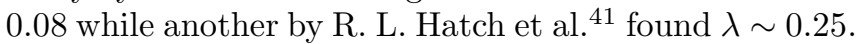
A more recent report ${ }^{45}$ gives $\sim 0.17$ with characteristic phonon energy $\sim 18 \mathrm{meV}$.

The results of X. Zhu et al. are further supported by an infrared study by A. D. LaForge et al $\stackrel{46}{*}$ which found a strong electron-phonon coupling to a $7.6 \mathrm{meV}$ optical phonon while S. Giraud et al. $\stackrel{47}{t}$ provide arguments for coupling to acoustic phonon with $\lambda \sim 0.42$ in their films and even larger in other geometries $\underline{48}$ Very recently J. A. Sobota et al. $\stackrel{49}{ }$, using time-resolved photoemission spectroscopy, find evidence for coupling to a $2.05 \mathrm{ThZ}$ surface phonon mode with the Dirac electrons in $\mathrm{Bi}_{2} \mathrm{Se}_{3}$. Possible complications in the interpretation of optical pumping on time resolved data were discussed by S. Ulstrup 50 and need to be kept in mind. Finally, the recent transport measurements of M.V. Costache et al. $\stackrel{51}{\underline{w}}$ were interpreted with strong coupling to a single optical phonon mode of energy $\sim 8 \mathrm{meV}$.

In this paper we will emphasize the effect of the 


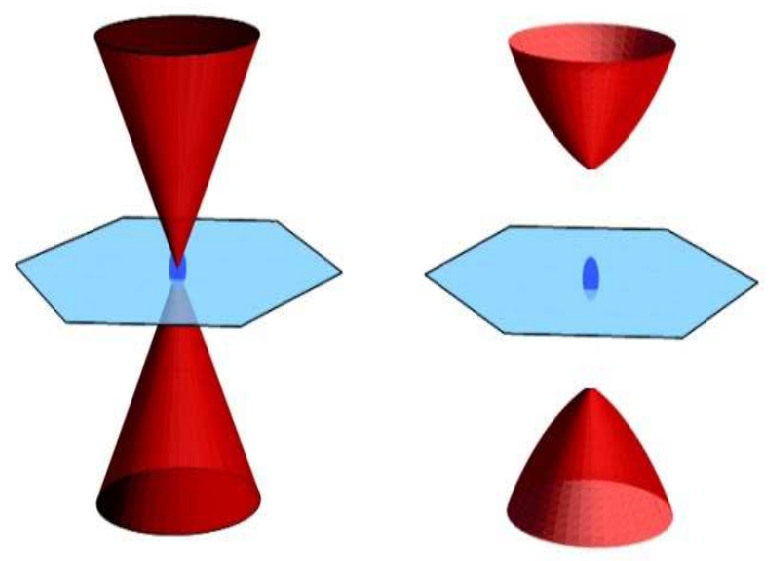

FIG. 1. (Color online) Illustrative representation of Dirac cones (left frame) and including a gap $\Delta$ (right frame).

electron-phonon interaction on the spin texture of gapped Dirac helical fermions. We will present for comparison, results for the familiar spectral density $\left(A_{I}(k, \omega)\right)$ associated with quasiparticle renormalization which we compared with the much less familiar equivalent function which enters gap renormalizations $\left(A_{z}(k, \omega)\right)$. This is the essential quantity for the calculation of the z-axis (perpendicular to the surface states) properties. While only $A_{I}(k, \omega)$ is needed in calculations of the density of states $N(\omega)$ both $A_{z}$ and $A_{I}$ enter the dynamic conductivity $\sigma_{x x}(\omega)$. In section II we present the necessary formalism for both the self energy associated with quasiparticle renormalizations $\Sigma_{I}(\omega)$ ) and with the renormalization of the gap $\left.\Sigma_{z}(\omega)\right)$ which determine the needed spectral functions $A_{I}(k, \omega)$ and $A_{z}(k, \omega)$. Numerical results are presented in section III which deals with spectral densities, density of states and optical conductivity. In section IV we consider both in plane and out of plane spin texture. A summary and conclusions are given in section V.

\section{FORMALISM}

We begin with a model hamiltonian for the electronic states at the surface of a topological insulator which has the form

$$
H_{0}=\hbar v_{F}\left[k_{x} \sigma_{y}-k_{y} \sigma_{x}\right]+\Delta \sigma_{z}+E_{0}(\mathbf{k})
$$

where $\sigma$ 's are Pauli spin matrices for real electron spin, $v_{F}$ is the Fermi velocity of the Dirac electrons, $\mathbf{k}$ is momentum, $\Delta$ is a gap which can be introduced for example by doping with magnetic impurities and $E_{0}(\mathbf{k})$ is a quadratic term $E_{0}(\mathbf{k})=\frac{\hbar^{2} k^{2}}{2 m}$ with $m$ the electron mass. The introduction of $E_{0}$ gives particle hole asymmetry.

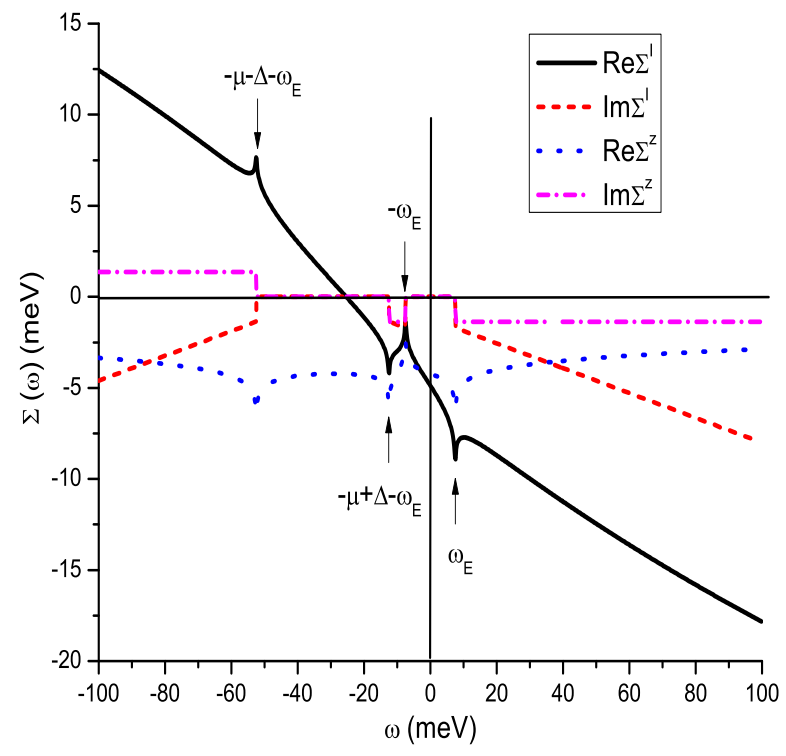

FIG. 2. (Color online) The real and imaginary part of the electron-phonon self energies $\Sigma^{I}(\omega)$ and $\Sigma^{z}(\omega)$ in meV as a function of $\omega$ in meV. $\Sigma^{I}(\omega)$ (real part, solid black), and imaginary part (dashed red) gives the quasiparticle renormalization and $\Sigma^{z}(\omega)$ (real part, dotted blue), and imaginary part (dash-dotted purple) gives the gap renormalization. Our choice of $\mathrm{g}=10$ corresponds to a mass enhancement $\lambda \approx 0.3$.

Here we will assume that $m$ is very large, as a first approximation, and will ignore this term.

The non interacting Green's function takes on the form

$$
G_{0}\left(\mathbf{k}, s, i \omega_{n}\right)=\frac{1}{i \omega_{n}+\mu-s \sqrt{\hbar^{2} v_{F}^{2} k^{2}+\Delta^{2}}}
$$

with $i \omega_{n}$ the Matsubara frequencies and $\mu$ is the non interacting chemical potential. The eigen energies are $\varepsilon_{k, s}=s \sqrt{\hbar^{2} v_{F}^{2} k^{2}+\Delta^{2}}$ with $s= \pm$ for conduction and valence band respectively. These dispersion curves are shown schematically in Fig. 1 with (right frame) and without (left frame) a gap.

The matrix $(2 \times 2)$ Green's function $\hat{G}_{0}\left(\mathbf{k}, i \omega_{n}\right)$ can be written in terms of the scalar $G_{0}\left(\mathbf{k}, s, i \omega_{n}\right)$ as

$$
\hat{G}_{0}\left(\mathbf{k}, i \omega_{n}\right)=\frac{1}{2} \sum_{s= \pm}\left(1+s \mathbf{F}_{\mathbf{k}} \cdot \sigma\right) G_{0}\left(\mathbf{k}, s, i \omega_{n}\right)
$$

with the vector $\mathbf{F}_{k}$ defined as

$$
\mathbf{F}_{\mathbf{k}}=\frac{\left(-\hbar v_{F} k_{y}, \hbar v_{F} k_{x}, \Delta\right)}{\sqrt{\hbar^{2} v_{F}^{2} k^{2}+\Delta^{2}}}
$$

We want to include an electron-phonon interaction. The simplest is the Holstein model for coupling to a phonon mode of energy $\omega_{E}$ with matrix element between electronic and phonon assumed to be a constant $(g)$. It is written as

$$
H_{e-p h}=-g \omega_{E} \sum_{\mathbf{k}, \mathbf{k}^{\prime}, s} c_{\mathbf{k}, s}^{\dagger} c_{\mathbf{k}^{\prime}, s}\left(b_{\mathbf{k}^{\prime}-\mathbf{k}}^{\dagger}+b_{\mathbf{k}-\mathbf{k}^{\prime}}\right)
$$


where $c_{\mathbf{k}, s}^{\dagger}$ creates an electron of momentum $\mathbf{k}$ and spin $s$ and $b_{\mathbf{k}^{\prime}-\mathbf{k}}^{\dagger}$ creates a phonon of momentum $\mathbf{k}^{\prime}-\mathbf{k}$ reduced to the first surface state Brillouin zone and energy $\omega_{E}$ with $g$ coupling constant. The perturbing Hamiltonian (5) provides a self energy to electron motion which has two components one proportional to the unit matrix $\widehat{I}$ and the other to $\widehat{\sigma}_{z}$. Writing $\widehat{\Sigma}\left(i \omega_{n}\right)=\Sigma^{I}\left(i \omega_{n}\right) \widehat{I}+$ $\Sigma^{Z}\left(i \omega_{n}\right) \widehat{\sigma}_{z}$ we get in lowest order perturbation theory

$$
\begin{aligned}
& \Sigma^{I}\left(i \omega_{n}\right)=\frac{g^{2} \omega_{E}^{2}}{2} \sum_{\mathbf{q}, s} \\
& {\left[\frac{f_{F}\left(\varepsilon_{q, s}\right)+f_{B}\left(\omega_{E}\right)}{i \omega_{n}+\mu+\omega_{E}-\varepsilon_{q, s}}+\frac{f_{B}\left(\omega_{E}\right)+1-f_{F}\left(\varepsilon_{q, s}\right)}{i \omega_{n}+\mu-\omega_{E}-\varepsilon_{q, s}}\right]}
\end{aligned}
$$

and

$$
\begin{aligned}
& \Sigma^{Z}\left(i \omega_{n}\right)=\frac{g^{2} \omega_{E}^{2}}{2} \sum_{\mathbf{q}, s} \frac{s \Delta}{\sqrt{\hbar^{2} v_{F}^{2} q^{2}+\Delta^{2}}} \times \\
& {\left[\frac{f_{F}\left(\varepsilon_{q, s}\right)+f_{B}\left(\omega_{E}\right)}{i \omega_{n}+\mu+\omega_{E}-\varepsilon_{q, s}}+\frac{f_{B}\left(\omega_{E}\right)+1-f_{F}\left(\varepsilon_{q, s}\right)}{i \omega_{n}+\mu-\omega_{E}-\varepsilon_{q, s}}\right]}
\end{aligned}
$$

where $f_{F}$ and $f_{B}$ are fermion and boson distribution functions $1 /\left[e^{(\omega-\mu) / k_{B} T} \pm 1\right]$ respectively. Here $\mu$ is the chemical potential which applies only to the electron distribution. Note that $\Sigma^{Z}\left(i \omega_{n}\right)$ in Eq. (7) is directly proportional to the gap $\Delta$ and will vanish for $\Delta=0$. In terms of the self energies $\Sigma^{I}\left(i \omega_{n}\right)$ and $\Sigma^{Z}\left(i \omega_{n}\right)$ the interacting matrix Green's function takes on the form

$$
\hat{G}\left(\mathbf{k}, i \omega_{n}\right)=\frac{1}{2} \sum_{s= \pm}\left(1+s \mathbf{H}_{\mathbf{k}} \cdot \sigma\right) G\left(k, s, i \omega_{n}\right)
$$

with

$$
\mathbf{H}_{\mathbf{k}}=\frac{\left(-\hbar v_{F} k_{y}, \hbar v_{F} k_{x}, \Delta+\Sigma^{Z}\left(i \omega_{n}\right)\right)}{\sqrt{\hbar^{2} v_{F}^{2} k^{2}+\left(\Delta+\Sigma^{Z}\left(i \omega_{n}\right)\right)^{2}}}
$$

and

$$
\begin{aligned}
& G\left(k, s, i \omega_{n}\right)= \\
& \frac{1}{i \omega_{n}+\mu-\Sigma^{I}\left(i \omega_{n}\right)-s \sqrt{\left(\Delta+\Sigma^{Z}\left(i \omega_{n}\right)\right)^{2}+\hbar^{2} v_{F}^{2} k^{2}}}(10)
\end{aligned}
$$

In Ref. (19) a factor $\Sigma^{Z *}\left(i \omega_{n}\right)$ was mistakenly introduced instead of $\Sigma^{Z}\left(i \omega_{n}\right)$ in (9) and (10). This leads to small numerical differences but has no qualitative significance. The spectral function associated with $\widehat{I}$ and $\widehat{\sigma}_{z}$ matrix are

$$
\begin{aligned}
A_{I}(k, s, \omega) & =-\frac{1}{\pi} \operatorname{Im} G\left(k, s, i \omega_{n} \rightarrow \omega+i \delta\right) \\
& \equiv A(k, s, \omega)
\end{aligned}
$$

which is the quantity measured in angular resolved photo emission spectroscopy ARPES ${ }^{35}$ Further

$$
\begin{aligned}
& A_{z}(k, s, \omega) \\
= & -\frac{1}{\pi} \operatorname{Im} \operatorname{Tr}\left[\sigma_{z} \hat{G}\left(k, s, i \omega_{n} \rightarrow \omega+i \delta\right)\right] \\
= & -\frac{1}{\pi} \operatorname{Im}\left\{\frac{s\left[\Delta+\Sigma^{Z}(\omega+i \delta)\right] G(k, s, \omega+i \delta)}{\sqrt{\hbar^{2} v_{F}^{2} k^{2}+\left(\Delta+\Sigma^{Z}(\omega+i \delta)\right)^{2}}}\right\} .
\end{aligned}
$$

A considerable and instructive mathematical simplification of these complicated expressions for (11) and (12) result when the imaginary part of the z-component of the self energy $\Sigma^{Z}\left(i \omega_{n} \rightarrow \omega+i \delta\right)$ is ignored. We get

$$
\begin{gathered}
A(k, s, \omega)=\frac{1}{\pi} \frac{\operatorname{Im} \Sigma^{I}(\omega)}{[\widetilde{\omega}-s \sqrt{M}]^{2}+\left[\operatorname{Im} \Sigma^{I}(\omega)\right]^{2}} \\
A_{z}(k, s, \omega) \\
=\frac{s\left[\Delta+\operatorname{Re} \Sigma^{Z}(\omega)\right] A(k, s, \omega)}{\sqrt{\hbar^{2} v_{F}^{2} k^{2}+\left(\Delta+\operatorname{Re} \Sigma^{Z}(\omega)\right)^{2}}}
\end{gathered}
$$

with

$$
\widetilde{\omega}=\omega+\mu-\operatorname{Re} \Sigma^{I}(\omega)
$$

and

$$
M=\hbar^{2} v_{F}^{2} k^{2}+\left(\Delta+\operatorname{Re} \Sigma^{Z}(\omega)\right)^{2}
$$

where $M$ is a function of $k$ and $\omega$. If we further take the imaginary part of $\Sigma^{I}(\omega)$ to be zero (13) reduces to a Dirac delta function but the real part of both $\Sigma^{I}$ and $\Sigma^{Z}$ remain which renormalize the single particle energies and the gap. The probability of occupation of the state $\mathbf{k}$ denoted by $n(k)$ at temperature $T$ is given by ${ }^{22}$

$$
n(k)=\int_{-\infty}^{+\infty} d \omega A(k, s, \omega) f(\omega)
$$

and this is to be compared with the corresponding expression for the z-component of spin $S_{z}(k)$

$$
S_{z}(k)=\int_{-\infty}^{+\infty} d \omega A_{z}(k, s, \omega) f(\omega) .
$$

We can also calculate the average value of the square root of the sum of the squares of $x$ and $y$ component of spin which remains locked perpendicular to momentum but its magnitude is changed by the electron-phonon coupling

$$
\sqrt{S_{x}^{2}(k)+S_{y}^{2}(k)}=\int_{-\infty}^{+\infty} d \omega A_{x-y}(k, s, \omega) f(\omega)
$$

with

$$
A_{x-y}(k, s, \omega)=-\frac{1}{\pi} \operatorname{Im}\left\{\frac{\left[\hbar v_{F} k\right] G(k, s, \omega)}{\sqrt{\hbar^{2} v_{F}^{2} k^{2}+\left(\Delta+\Sigma^{Z}(\omega)\right)^{2}}}\right\}
$$

The density of electronic states $N(\omega)$ follows from $A(k, s, \omega)$ on integration over $k$

$$
N(\omega)=\sum_{\mathbf{k}, s} A(k, s, \omega)
$$

This quantity enters scanning tunneling microscopy experiments (STM) $\stackrel{32-36}{2}$ The real part of the dynamic longitudinal conductivity which gives the absorption spectrum for light follows as 25

$$
\begin{aligned}
& \operatorname{Re}_{x x}(\omega)=\frac{e^{2} v_{F}^{2} \pi^{2}}{\omega} \sum_{\mathbf{k}} \int_{-\infty}^{\infty} \frac{d \omega}{2 \pi}[f(\omega)-f(\omega+\Omega)] \\
& \times\left[A_{I}(\mathbf{k}, \omega) A_{I}(\mathbf{k}, \omega+\Omega)-A_{z}(\mathbf{k}, \omega) A_{z}(\mathbf{k}, \omega+\Omega)\right]
\end{aligned}
$$


with $A_{I}(\mathbf{k}, \omega)=\sum_{s} A_{I}(k, s, \omega)$ and $A_{z}(\mathbf{k}, \omega)=$ $\sum_{s} A_{z}(k, s, \omega)$. Note that both $A_{z}$ and $A_{I}$ enter this quantity even though it is an in-plane property.

\section{NUMERICAL RESULTS FOR SELF ENERGY AND DERIVED QUANTITIES}

In lowest order perturbation theory the chemical potential $\mu$ in Eq. (6) and (7) is to be interpreted as having its non interacting, independent particle value and will be quoted in all the results to be presented in this paper. With electron-phonon coupling the chemical potential will in general be shifted from its free band value to $\mu_{\text {interacting }}=\mu+\operatorname{Re} \Sigma^{I}\left(i \omega_{n} \rightarrow \omega+i \delta\right)$. In Fig. 2 we show our results for $\Sigma\left(i \omega_{n} \rightarrow \omega+i \delta\right)$ in meV as a function of energy $\omega$ in meV where we have analytically continued from Matsubara to real frequencies. The solid black curve is $\operatorname{Re} \Sigma^{I}$ with $\operatorname{Im} \Sigma^{I}$ given by the dashed red curve while the dotted blue is for $\operatorname{Re}^{Z}(\omega)$ with its imaginary part $\operatorname{Im} \Sigma^{Z}(\omega)$ given by the purple dash-dotted curve. First the real part of the quasiparticle self energy is not zero at $\omega \simeq 0$ but rather is of the order of $\simeq-5 \mathrm{meV}$ which reduces the chemical potential of the interacting picture by about $1 / 4$ of its bare band value here taken to be $25 \mathrm{meV}$ with the gap $\Delta$ equal to $20 \mathrm{meV}$. With $g$ set to a value of 10 in Eq. (6) and (7), the mass enhancement parameter $\lambda \sim 0.3$ as can be seen in Fig. 2. The parameter $\lambda$ is the slope of the real part of $\Sigma^{I}(\omega)$ at $\omega=0$, i.e. $\lambda=(d / d \omega) \operatorname{Re} \Sigma^{I}(\omega)$ at $\omega=0$. We have also taken the Einstein phonon energy to be $7.5 \mathrm{meV}$ which is representative of what is seen in many but not all experiments in $B i_{2} S_{3}$ and has been identified in the work of X. Zhu et al $\underline{42}$ as a surface phonon. As discussed in the introduction coupling to specific optical phonons have been found in other experiments $41.43-46$. Of course there can also be coupling to acoustic phonons as studied by Giraud et al. $\underline{47.48}$ These authors use a continuum model for the phonons and a deformation potential model to describe their coupling to the helical surface Dirac fermions with due attention to the modifications brought about by helicity of the charge carriers. In our simplified model these complications enter only in determining the size of $g$ in Eq. (6) and (7) which we set through consideration of experiment (i.e. $\lambda \sim 0.3$ ). In principle these could also be coupling to electronic modes but this is not considered in this work which deals with electron-phonon coupling alone. Our choice of gap $\Delta=10 \mathrm{mev}$ is representative of the work of Chen et al $\stackrel{9}{=}$ who found in ARPES experiments a gap of $\simeq-7 \mathrm{mev}$ in $B i_{2} S e_{3}$ magnetically dopped with $M n$. We note prominent phonon structure at $\omega= \pm \omega_{E}= \pm 7.5 \mathrm{meV}$ by choice, with additional structures at $-\mu+\Delta-\omega_{E}$ and at $-\mu-\Delta-\omega_{E}$. For $\omega>0$ the imaginary part of the quasiparticle self energy is zero in the interval $\left(0, \omega_{E}\right)$ after which its absolute magnitude is finite and increases linearly with increasing value of $\omega$. This dependence reflects the linear dependence on energy of the underlying bare density of states. Also it needs to
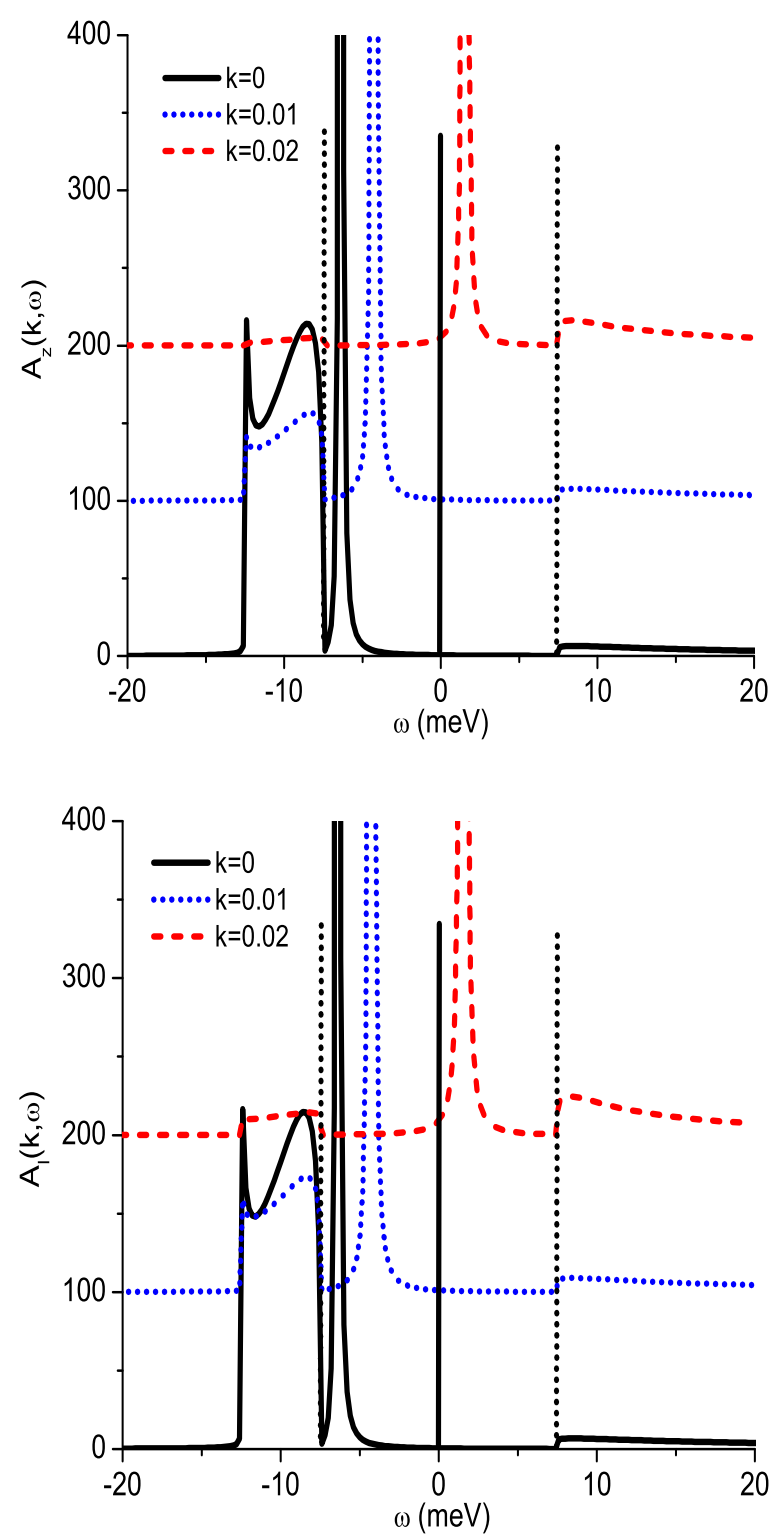

FIG. 3. (Color online) The spectral density $A(k, \omega)$ as a function of energy $\omega$ in meV for three values of momentum $k$ namely $k=0$ (solid black), $k=0.01$ (dotted blue) and $k=0.02$ (dashed red) in units of the inverse lattice parameter $a$. The top frame is for $A_{z}(k, \omega)$ (associated with the gap renormalization) and the bottom frame is for $A_{I}(k, \omega)$ associated with quasiparticle renormalization. Vertical dotted black lines are at $\omega= \pm \omega_{E}$ with phonon Einstein energy set at $7.5 \mathrm{meV}$. The gap $\Delta=20 \mathrm{meV}$ and the bare chemical potential is $\mu=25 \mathrm{meV}$.

be negative because $-\operatorname{Im} \Sigma^{I}\left(i \omega_{n} \rightarrow \omega+i \delta\right)$ is a scattering rate, and hence positive. By contrast the imaginary part of the gap self energy is flat because the extra factor of $\Delta / \sqrt{\hbar^{2} v_{F}^{2} q^{2}+\Delta^{2}}$ appearing in (7) but not in (6) compensates for the density of states variarion which comes from the sum over $q$. Note also that both self energies 


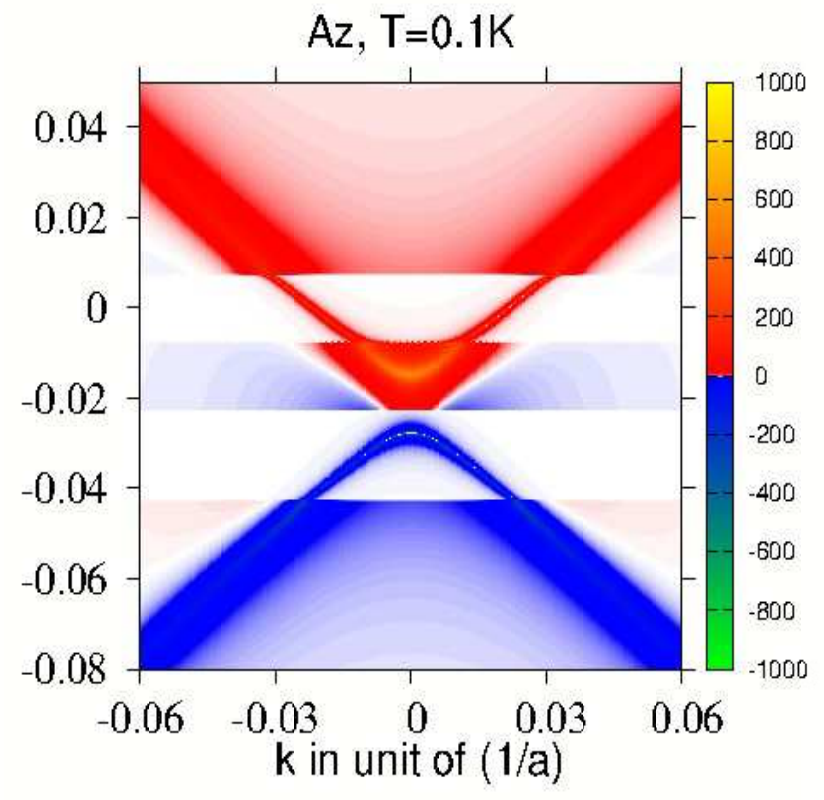

FIG. 4. (Color online) A color plot of the gap spectral density $A_{z}(k, \omega)$ as a function of energy $\omega$ in $(\mathrm{eV})$ vertical axis and momentum $k$ in $(1 / a)$ horizontal axis, with $a$ the lattice parameter. The gap $\Delta=10 \mathrm{meV}$ and the Einstein phonon energy $\omega_{E}=7.5 \mathrm{meV}$.

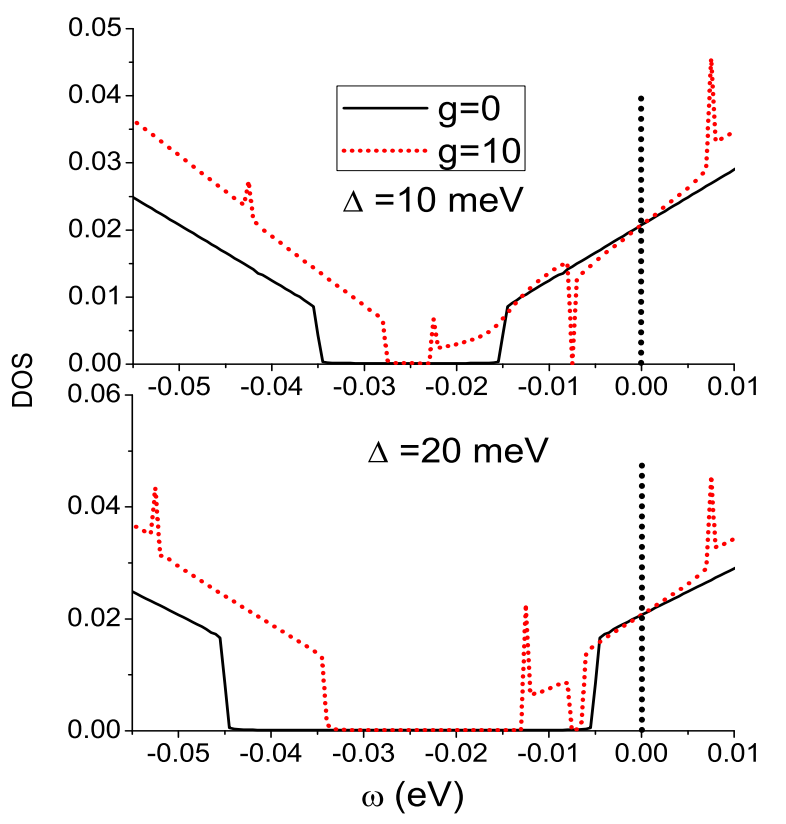

FIG. 5. (Color online) The density of states $N(\omega)$ in inverse $\mathrm{eV}$ versus energy $\omega$ in $\mathrm{eV}$. The dotted red line includes the electron-phonon interaction with $g=10$ in Eq. (6)-(7). The solid black line which is for comparison is for bare bands $(g=$ 0 ). The top frame has a gap $\Delta=10 \mathrm{meV}$ while the bottom frame has a gap of $20 \mathrm{meV}$.

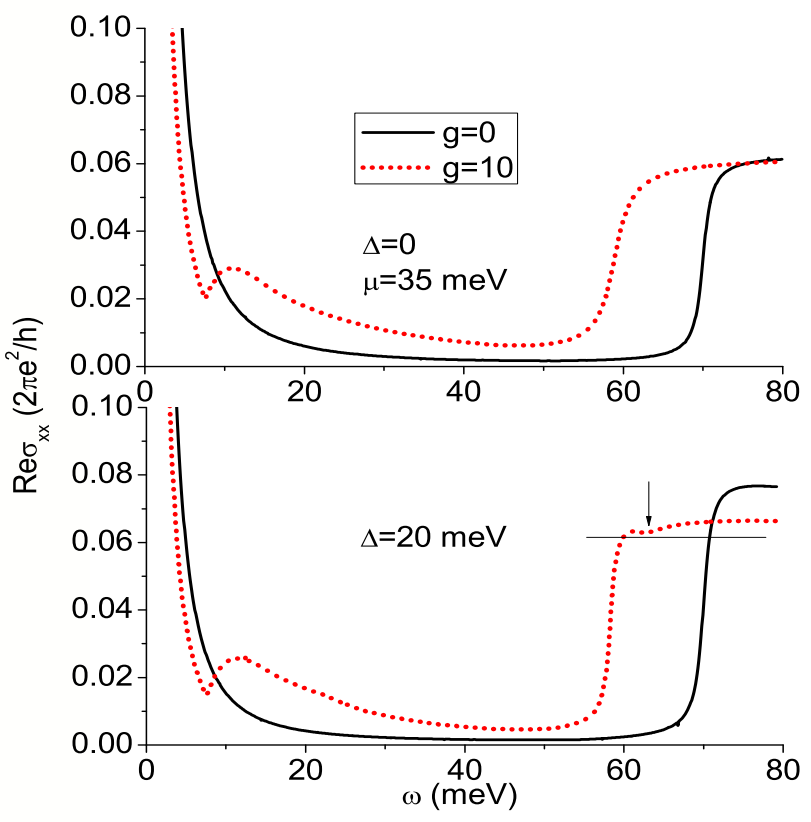

FIG. 6. (Color online) The real part of the longitudinal dynamic optical conductivity $\operatorname{Re} \sigma_{x x}(\omega)$ in units of $e^{2} / \hbar$ as a function of photon energy $\omega$ in $\mathrm{meV}$. The dotted red line includes the electron-phonon interaction with $g=10$ in Eq. (6)-(7). The solid black line, which is for comparison, is for bare bands $(g=0)$. The top frame has no gap $\Delta=0$ and the bottom frame includes a gap $\Delta=20 \mathrm{meV}$. In both cases the bare chemical potential $\mu=35 \mathrm{meV}$. The arrow identifies the phonon structure.

have a finite imaginary part in the interval $\omega \in\left(0,-\omega_{E}\right)$ and $\omega<-\mu-\Delta-\omega_{E}$. While the real part of the quasiparticle self energy can be both positive or negative, the real part of the gap self energy is everywhere negative and so decreases the bare gap $\Delta$ at all $\omega$ considered here. Its magnitude is everywhere of order $1 / 4$ of the input value of $\Delta$ with some $\omega$ dependence and is encoded with boson structure. These self energies have a profound effect on the corresponding spectral densities of Eq. (11) and (12) as we see in Fig. 3 where the top frame applies to $A_{z}(k, \omega)=A_{z}(k, s>0, \omega)$ (conduction band) and the bottom frame is for $A_{I}(k, \omega)=A_{I}(k, s>0, \omega)$. For the range of values of $k$ shown namely, $k=0.0,0.01$ and 0.02 in units of the inverse of the lattice parameter $(a)$, these two functions do not differ much from each other. This is traced to the fact that $A_{z}(k, \omega)$ has the extra factor $\frac{\Delta}{\sqrt{\hbar^{2} v_{F}^{2} k^{2}+\Delta^{2}}}$ as compared with $A_{I}(k, \omega)$ where we have, for simplicity, neglected renormalizations as these do not change the argument in an important way. As long as $\hbar v_{F} k$ is small compared with $\Delta$ the relevant additional factor is near one. For large $k$ however, larger than those shown in Fig. 3, $A_{z}(k, \omega)$ will become much smaller in magnitude than $A_{I}(k, \omega)$ by a factor of $\Delta /\left(\hbar v_{F} k\right)$. Returning to the top frame of Fig. 3 we first note in the solid black curve for $k=0$ (which is the momentum of the bottom of the bare conduction band) a large, only 
slightly broaden and shifted in energy, quasiparticle peak centered approximately at $\simeq 6 \mathrm{meV}$ below the Fermi surface at $\omega \simeq 0$. For the bare bands it would be at $5 \mathrm{meV}$ instead. But with correlations there are also additional features. There is a large increase in $A_{z}(k, \omega)$ starting at $\omega=-\omega_{E}=-7.5 \mathrm{meV}$ followed by a large boson structure extending down to $\simeq-12.5 \mathrm{meV}$ where it drops to zero. Such a feature is also seen in the dashed red curve but it is much smaller. The lower cut off is identified to correspond to the energy $-\mu-\omega_{E}+\Delta$. If we had shown the valence band contribution to the spectral density it would have a further boson structure at $-\mu-\omega_{E}-\Delta$ as in the self energy of Fig. 2. It is clear from this description that the effect of the electron-phonon interaction on the bare band is much more complicated than a simple constant shift in gap value and a slight shift in quasiparticle energies with small broadening. Note also the clear phonon sidebands at energies above $\omega=\omega_{E}$ in all three curves. The black vertical dotted lines identify energies $\omega= \pm \omega_{E}$ for easy reference. A different representation of these changes is given in Fig. 4 where we show a false color plot of $A_{z}(k, \omega)$ as a function for energy $\omega$ along the vertical axis and momentum $k$ along the horizontal. As seen in Eq. (12) and (14), $A_{z}$ carries the sign of $s$ and is positive (red) in the renormalized conduction band and negative (blue) in the renormalized valence band. The phonon at $\omega= \pm \omega_{E}$ is clearly identified as is the bottom of the renormalized conduction band at $-\mu-\omega_{E}+\Delta$ and the valence band phonon structure at $-\mu-\omega_{E}-\Delta$. Particularly striking is the large modification of the bare band dispersion curves in the region just above the renormalized conduction band minimum.

The large renormalization effects seen in Fig. 3 and Fig. 4 can have a profound effect on certain quantities while at the same time have much more modest manifestations in others as we will now describe. In Fig. 5 we show results for the density of states (DOS) $N(\omega)$ given in Eq. (21). The top frame is for a gap $\Delta=10 \mathrm{meV}$ and the bottom for $\Delta=20 \mathrm{meV}$. The red dotted curve is the renormalized quasiparticle DOS while the black solid curve is the bare band case shown for comparison. In both frames the bare chemical potential is set at 25 $\mathrm{meV}$, which is $5 \mathrm{meV}$ above the gap in the conduction band (for the case $\Delta=20 \mathrm{meV}$ ). The energy variable $\omega$ is set so that $\omega=0$ corresponds to the Fermi surface so that the bottom of the conduction band is at -5 $\mathrm{meV}$ in bottom frame and $-15 \mathrm{meV}$ in top frame with the top of the valence band at $-45 \mathrm{meV}$ and $-35 \mathrm{meV}$ respectively. In both cases the remaining gap between valence and conduction band has been very much reduced over its bare band value. The four phonon structures at $\omega= \pm \omega_{E},-\mu-\omega_{E}-\Delta$ and $-\mu-\omega_{E}+\Delta$ are clearly seen with the bottom of the conduction band given by the energy $-\mu-\omega_{E}+\Delta$. By comparison, the corresponding boson structures in the real part of the dynamic longitudinal optical conductivity $\operatorname{Re} \sigma_{x x}(\omega)$ which is more closely related to a convolution of two DOS factors and is given in Eq. (22), are much more modest as seen in Fig. 6.
The top frame is for gapless Dirac fermions $(\Delta=0)$ and is included for comparison while the bottom frame is for the gapped case with $\Delta=20 \mathrm{meV}$. The solid black curves are for the bare band case with a small residual scattering rate included to broaden out the Drude peak due to intraband transitions. This peak is large only at small $\omega$ and is centered at $\omega=0$. The bare chemical potential is $35 \mathrm{meV}$ and we see the onset of a second absorption band coming from the interband transitions which start at $\omega=2 \mu$. These transitions continue up to large energies and provide the so called universal background. In our units for $\operatorname{Re} \sigma_{x x}(\omega)$ which is $e^{2} / \hbar$, this background 39.40 has a height of $1 / 16$. As is clear in the top frame for $\Delta=0$ this height is almost unaffected by the electron-phonon interaction (red dotted curve). Staying with the top frame we see however the appearance of the Holstein processes above $\omega=\omega_{E}$ which provides significant phonon assisted absorption in the photon region above $\omega_{E}$ and below the main interband absorption edge at $2 \mu$. The other feature to be noted is that, for the correlated case, the onset of the interband transitions has moved to lower energies and is now at twice the value of the interacting chemical potential. The lower frame which is for the gap fermion case has another important element. As is well known, $19,39,40$ when $\Delta \neq 0$ there is a peak in the interband transitions just above the threshold energy which persists up to a few $\Delta$ above the threshold before the value of the background is reestablished at its universal value. In the clean limit we have the analytic result

$$
\begin{aligned}
\operatorname{Re} \sigma_{x x}(\omega)= & \frac{e^{2}}{4 \hbar}\left[\delta(\omega) \frac{\mu^{2}-\Delta^{2}}{|\mu|} \theta\left(\mu^{2}-\Delta^{2}\right)+\right. \\
& \left.\frac{\omega^{2}+4 \Delta^{2}}{4 \omega^{2}} \theta(\omega-2 \max (|\mu|, \Delta))\right]
\end{aligned}
$$

where $\theta$ is a heaviside function. We see that, just above the interband onset the conductivity is larger than its universal value of $\frac{e^{2}}{16 \hbar}$ as is seen most clearly in the solid black curve of the lower frame of Fig. 6 which is the bare band result. The horizontal straight line segment indicate $1 / 16$. We note that, with electron-phonon (dotted red curve), the magnitude of the absorption in the region of the edge still remains above the universal background value but now there is also a small phonon structure highlighted by the vertical arrow. No such structure is seen in the top frame for $\Delta=0$. It is the variation with energy of the background (in the presence of a finite gap) which allows for the phonon structure to be revealed.

\section{SPIN TEXTURE}

The z-axis spin texture is determined from the spectral density $A_{z}(k, s, \omega)$ of Eq. (12). Results are presented in Fig. 7 for $S_{z}(k)$ as a function of momentum $k$ normalized to its value at the Fermi surface $\left(k_{F}\right) . S_{z}(k)$ involves an integral over frequency $\omega$ of the overlap of $A_{z}(k, s, \omega)$ and the Fermi function $f(\omega)$ as given in Eq. (18). We only 


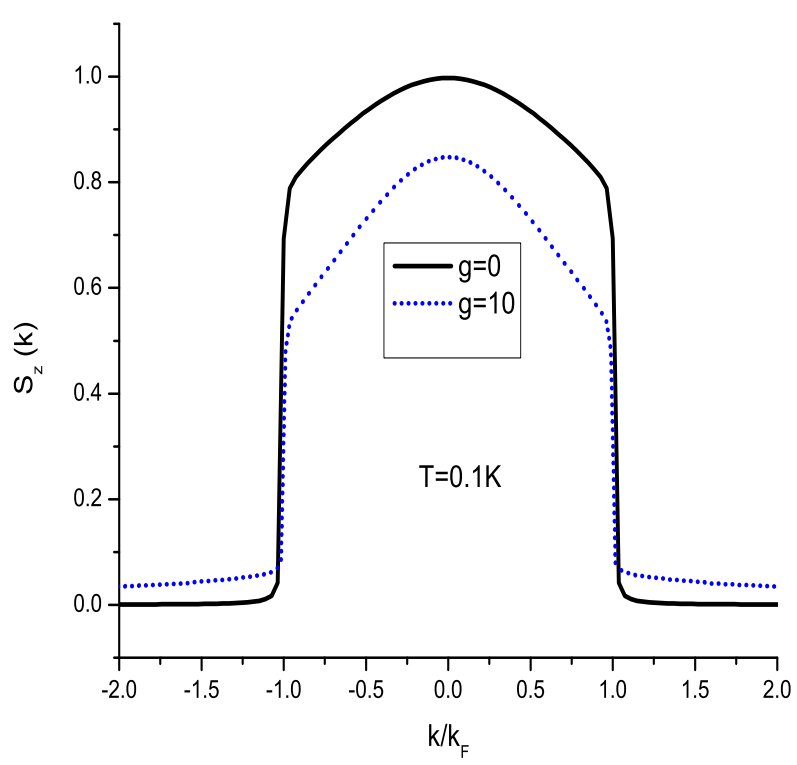

FIG. 7. (Color online)The z-component of spin $S_{z}(k)$ as a function of momentum $k$ normalized to $k_{F}$. The solid black line is for $g=0$ (no electron-phonon interaction) and is for comparison with the dotted blue curve which includes the self energy with coupling $g=10$ (Eq. (6)-(7)).

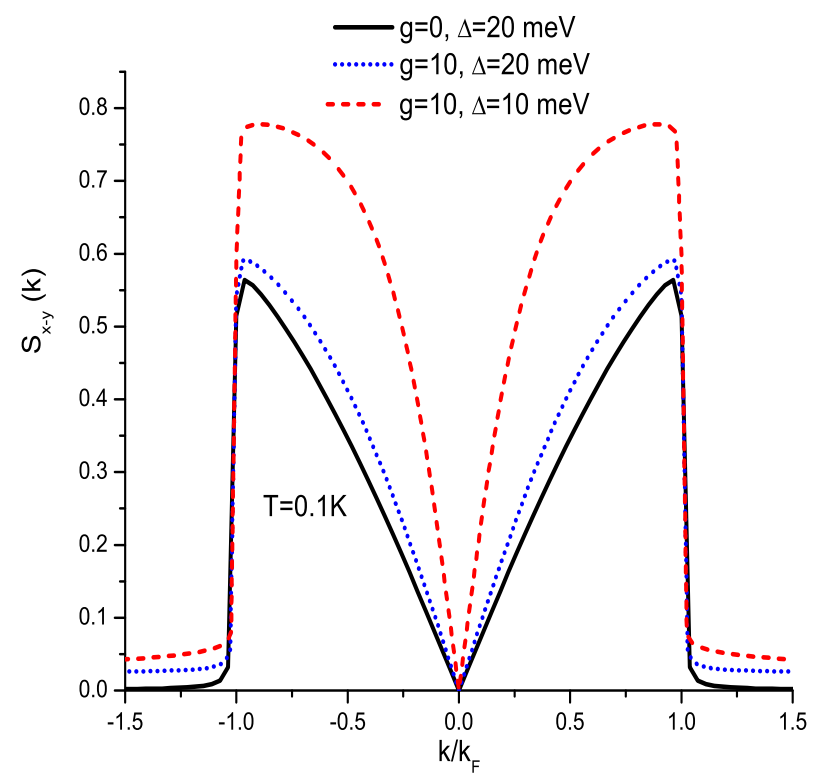

FIG. 8. (Color online) The magnitude of the in plane spin $S_{x-y}(k)$ as a function of momentum $k$ normalized to its value at the Fermi surface $k_{F}$. The solid black curve is for comparison and involves the bare bands $(g=0)$ with gap $\Delta=20$ $\mathrm{meV}$. The dotted blue has the same gap but includes the electron-phonon renormalizations with coupling $g=10$ in Eq. (6)-(7). The dashed red is for a smaller value of gap $\Delta=10$ meV.

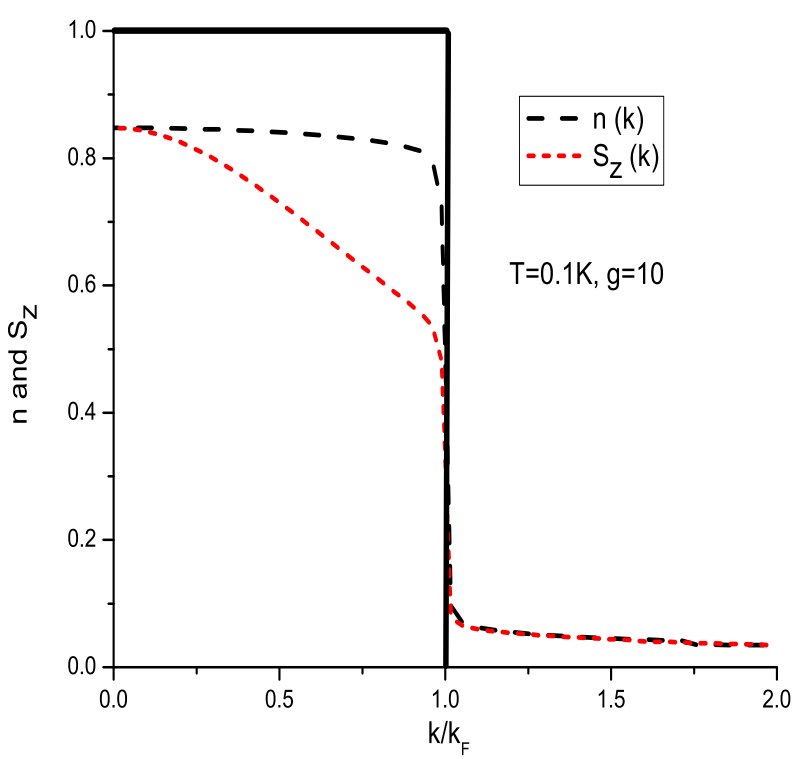

FIG. 9. (Color online) Comparison of the z-component of spin $S_{z}(k)$ of Eq. (18) as a function of momentum $k$ normalized to its value at the Fermi surface $k_{F}$ (short dashed red curve) with the probability of occupation of state $n(k)$ of Eq. (17) (long dashed black curve). The heavy solid black line gives the case of bare bands for comparison.

show results for the conduction band $s=+$. For the valence band $s=-1$ and $S_{z}(k)$ will change sign. The gap $\Delta=20 \mathrm{meV}$ and the chemical potential $\mu=25 \mathrm{meV}$ as before in section III. The solid black curve is the bare band result for temperature $T=0.1 K$. We see an abrupt drop to zero at $k / k_{F}=1$ because of the thermal factor which here is basically a Heaviside function with cut off at the Fermi surface. By contrast the dotted blue curve includes the electron-phonon renormalization discussed at length in the previous section. Now the magnitude of $S_{z}(k)$ at $k=0$ is reduced below its bare band value. More importantly the jump at $k / k_{F}$ is smaller than it is in the solid curve and further, there are finite tails beyond this momentum which are entirely due to many body correlation effects that go beyond a bare band description. They can be taken as representative of other correlation effect such as those due to electron-electron interactions rather than electron-phonon. Compared to the modifications found in the spectral densities, the density of states and the conductivity, these effects are certainly not as spectacular. Mathematically this results from the fact that $S_{z}(k)$ involves an integration over energy $\omega$ while it is this energy dependence which reflects most directly the details of the correlation effects.

The in-plane spin texture is also changed, although the perpendicular spin momentum locking remains. In Fig. 8 we show results for the momentum dependence (in units of $\left.k / k_{F}\right)$ of $S_{x-y}(k)$ defined in Eq. (19). It gives the magnitude of the in-plane spin at momentum $k$. Without 
interactions it would be equal to $\frac{\hbar v_{F} k}{\sqrt{\hbar^{2} v_{F}^{2} k^{2}+\Delta^{2}}} \theta\left(k_{F}-k\right)$ and is shown as the solid black curve for a gap of $20 \mathrm{meV}$ and temperature $T=0.1 \mathrm{~K}$. It starts at zero for $k=0$ and rises monotonically with a sharp cut off at $k / k_{F}=1$ at zero temperature. When the electron-phonon interaction is included through Eq. (20) we get the dotted blue curve which follows closely the solid black curve with the important difference that it has tails beyond $k=k_{F}$ characteristic of correlation effects. The dashed red curve is for the same value of $g$ but now the gap has been reduced to $10 \mathrm{meV}$. In this case the curve rises more sharply out of $k=0$, flattens before showing a sharp drop at $k=k_{F}$ and the correlation tails beyond this drop off are now considerably larger. The correlation tails seen in $S_{z}(k)$ as well as in $S_{x-y}(k)$ are very similar to the much more familiar $\operatorname{case}^{22}$ of the momentum distribution in a fermion system with electron-phonon interaction. The probability of occupation of the state $k$ at zero temperature denoted by $n(k)$ is given in Eq. (17) and in the bare band picture is a step function $\theta\left(k_{F}-k\right)$. As shown in Fig. 9 long dashed black curve for $T=0.1 \mathrm{~K}, g=10$ and a gap $\Delta=20 \mathrm{meV}, n(k)$ is considerably reduced from value one throughout the occupied states. It still has a finite discontinuous jump at $k=k_{F}$ with correlation tail for $k>k_{F}$. The short red dashed curve is $S_{z}(k)$ previously calculated and presented here for comparison with $n(k)$. This shows the qualitatively similar effect of the electron-phonon interaction on these two quantities.

\section{CONCLUSIONS}

We calculated the effect of electron-phonon coupling on a system of helical gapped Dirac fermions. A simple Holstein model with coupling to a single Einstein phonon of energy $\omega_{E}$ was used. For massive Dirac fermions two self energies need to be introduced. There is the familiar quasiparticle renormalizarion which changes the bare band energies to dressed dispersion curves and provides damping. In addition there is a second self energy directly associated with modifications of the gap. It is energy $(\omega)$ dependent and complex with the real part directly modulating the magnitude of the bare band gap. Both these self energies enter the quasipaticle spectral density $A(k, s, \omega)$ with $k$ momentum $\omega$ energy and $s= \pm$ giving conduction and valence band respectively. In direct analogy, a second spectral density $A_{z}(k, s, \omega)$ associated with the gap channel is also introduced. The first function $A(k, s, \omega)$ can be measured directly in angular resolved photo-emission spectroscopy (ARPES). Its average over momentum $k$ determines the electronic density of states $N(\omega)$ measured in scanning tunneling spectroscopy (STM). The second $A_{z}(k, s, \omega)$ enters along with $A(k, s, \omega)$ the expressions for the dynamic longitudinal optical conductivity. It also determines the out of plane spin texture. Both $A$ and $A_{z}$ are functions of $\omega$ and are encoded with sharp phonon structures at $\omega= \pm \omega_{E},-\mu-\omega_{E}-\Delta$ and $-\mu-\omega_{E}+\Delta$. These phonon structures manifest directly in the density of states and in a somewhat different way in the optical conductivity.

The out of plane and in plane spin textures are modified by the electron-phonon interaction. But these quantities involve an integration over energy of an overlap of spectral density and thermal factor. Consequently the manifestation of phonon-electron coupling in these quantities is more subtle and not as direct. Nevertheless important corrections to a bare band picture arise. Just as the probability of occupation of a state of momentum $k$ is reduced from one for $k$ less than $k_{F}$, and extended (in momentum) tails appear for $k>k_{F}$, the magnitude of $S_{z}(k)$ follows the same trend. A similar picture applies for the magnitude of the in plane spin component. While the spin remains locked in the direction perpendicular to momentum, correlation tails appear beyond $k=k_{F}$. While the calculations are for the explicit case of the electron-phonon interaction, they serve to illustrate how many body interactions in general modify the bare band picture.

\section{ACKNOWLEDGMENTS}

This work was supported by the Natural Sciences and Engineering Research Council of Canada (NSERC) and the Canadian Institute for Advanced Research (CIFAR). This material is also based upon work supported by the National Science Foundation under the NSF EPSCoR Cooperative Agreement No. EPS-1003897 with additional support from the Louisiana Board of Regents. JPC initiated the work and wrote much of the text, ZL did all the calculations.

\section{REFERENCES}

* lizhou@mcmaster.ca

† carbotte@mcmaster.ca

1 M. Z. Hasan and C. L. Kane, Rev. Mod. Phys. 82, 3045 (2010).

2 X.-L. Qi and S.-C. Zhang, Rev. Mod. Phys. 83, 1057 (2011).
3 J. Moore, Nature (London) 464, 194 (2010).

4 D. Hsieh et al., Nature (London),452, 970 (2008).

5 Y. L. Chen, J. G. Analytis et al., Science 325, 178(2009).

6 D. Hsieh et al., Nature (London),460, 1101 (2009).

7 C. Jozwiak, X. L. Chen et al., Phys. Rev. B 84, 165113 (2011). 
8 S.-Y. Xu, X Xia et al., Science 332, 560 (2011).

9 Y. L. Chen, J.-H. Chu et al., Science 329, 659 (2010).

10 H.-Z. Lu, W.-Y. Shan et al., Phys. Rev. B 81, 115407 (2010).

11 J. Linder, T. Yokoyama and A. Sudbø, Phys. Rev. B 80, 205401 (2009).

12 K. F. Mak, C. Lee, J. Hone, J. Sham and T. F. Heinz, Phys. Rev. Lett. 105, 136805 (2010).

13 A. Splendiani, L. Sun et al., Nano Lett. 10, 1271 (2010).

14 C. Lee et al., ACS Nano 4, 2695 (2010).

15 W. Feng, Y. Yao, W. Zhu, J. Zhou, W. Yao and D. Xiao, Phys. Rev. B. 86, 165108 (2012).

16 Zhou Li and J. P. Carbotte, Phys. Rev. B. 86, 205425 (2012).

17 B. Aufray, A. Kara, S. Vizzini, H. Oughaddou, C. Léandri, B. Ealet and G. Lay, Appl. Phys. Lett. 96, 183102(2010).

18 P. De Padova, C. Quaresima, C. Ottaviani, P. M. Sheverdyaeva, P. Moras, C. Carbone, D. Topwal, B. Olivieri, A. Kara, H. Oughaddou, B. Aufray, and G. Lay, Appl. Phys. Lett. 96, 261905 (2010).

19 L. Stille, C. J. Tabert and E. J. Nicol, Phys. Rev. B 86, 195405 (2012).

20 M. Ezawa, Phys. Rev. Lett. 110, 026603 (2013).

21 M. Ezawa, Phys. Rev. Lett. 109, 055502 (2012).

22 J. P. Carbotte, E. J. Nicol and S. G. Sharapov, Phys. Rev. B 81, 045419 (2010).

23 Zhou Li and J. P. Carbotte, Phys. Rev. B. 88, 195133 (2013)

24 Zhou Li and J. P. Carbotte, Phys. Rev. B. 88, 045417 (2013).

25 Zhou Li and J. P. Carbotte, Physica B 421, 97 (2013), DOI: $10.1016 /$ j.physb.2013.04.030.

${ }^{26}$ T. Stauber and N. M. R. Peres, J. Phys.: Condens. Matter 20, 055002 (2008).

27 A. Pound, J. P. Carbotte and E. J. Nicol, Phys. Rev. B. 85, 125422 (2012).

28 Ion Garate, Phys. Rev. Lett. 110, 046402 (2013).

29 J. P. F. Leblanc, J. Hwang and J. P. Carbotte, Phys. Rev. B 85, 115126 (2012).

30 J. P. F. Leblanc, J. P. Carbotte and E. J. Nicol, Phys. Rev.
B 84, 165448 (2011)

31 A. Pound, J. P. Carbotte and E. J. Nicol, Phys. Rev. B. 84, 085125 (2011).

32 D. L. Miller et al., Science 324, 924 (2009).

${ }^{33}$ G. Li, A. Luican and E. Y. Andrei, Phys. Rev. Lett. 102, 176804 (2009).

34 E. J. Nicol and J. P. Carbotte, Phys. Rev. B 80, 081415 (2009).

35 S. Y. Zhou et al., Phys. Rev. B 78, 193404 (2008).

36 A. Pound, J. P. Carbotte and E. J. Nicol, Euro. Phys. Lett. 94, 57006 (2011).

37 Z. Li et al., Nature Phys. 4, 532 (2008).

38 J. P. Carbotte, J. P. F. Leblanc and P. E. C. Ashby, Phys. Rev. B. 87, 045405 (2013).

39 V. P. Gusynin, S. G. Sharapov and J. P. Carbotte, Phys. Rev. Lett. 96, 256802 (2006).

40 V. P. Gusynin, S. G. Sharapov and J. P. Carbotte, New J. Phys. 11, 095013 (2009).

41 R. C. Hatch et al. Phys. Rev. B 83, 241303 (2011).

42 Xuetao Zhu, L. Santos, R. Sankar, S. Chikara, C. . Howard, F. C. Chou, C. Chamon, and M. El-Batanouny, Phys. Rev. Lett. 107, 186102 (2011);

43 Xuetao Zhu, L. Santos, C. Howard, R. Sankar, F. C. Chou, C. Chamon, and M. El-Batanouny, Phys. Rev. Lett. 108, 185501 (2012).

44 Z.-H. Pan, A. V. Fedorov, D. Gardner, Y. S. Lee, S. Chu, and T. Valla, Phys. Rev. Lett. 108, 187001 (2012).

45 C. Chen et al. Scientific Reports 3, 2411 (2013).

46 A. D. LaForge, A. Frenzel, B. C. Pursley, Tao Lin, Xinfei Liu, Jing Shi, and D. N. Basov, Phys. Rev. B 81, 125120 (2010).

47 S. Giraud, A. Kundu, and Reinhold Egger, Phys. Rev. B. 85, 035441 (2012).

48 S. Giraud and Reinhold Egger, Phys. Rev. B. 83, 245322 (2011).

49 Jonathan A. Sobota et al., Arxiv: 1410.3437 (2014).

50 S. Ulstrup et al., Arxiv: 1502.01933 (2015).

51 M.V. Costache, I. Neumann, J.F. Sierra, V. Marinova, M.M. Gospodinov, S. Roche, and S.O. Valenzuela, Phys. Rev. Lett. 112, 086601 (2014). 